Punjab University Journal of Mathematics (2021),53(9),679-690

https://doi.org/10.52280/pujm.2021.530905

\title{
Some Smarandache Curves Constructed from a Spacelike Salkowski Curve with Timelike Principal Normal
}

\author{
Sleyman enyurt ${ }^{1 *}$, Kemal Eren ${ }^{2}$ \\ ${ }^{1}$ Department of Mathematics, Faculty of Arts and Sciences \\ Ordu University, Ordu, Turkey, \\ Corresponding author Email:ssenyurt@odu.edu.tr \\ ${ }^{2}$ Department of Mathematics, Faculty of Arts and Sciences \\ Sakarya University, Sakarya, Turkey, \\ Email: kemaleren52@gmail.com
}

Received: 12 November, 2019 / Accepted: 12 July, 2021 / Published online: 25 September, 2021

\begin{abstract}
In this article, we investigate the regular Smarandache curves constructed from the Frenet vectors of spacelike Salkowski curve with a timelike principal normal. In the first part of the study, literature research was conducted. In the second part, general information about the curve and spacelike Salkowski curve in Minkowski space are given. In the last part, the Frenet apparatus of the Smarandache curves are calculated. We draw a graphic of the obtained Smarandache curves and some related results about Smarandache curves are given.
\end{abstract}

\section{AMS (MOS) Subject Classification Codes: 53A04}

Key Words: Minkowski space, spacelike Salkowski curve, spacelike Smarandache curve.

\section{INTRODUCTION}

Curves are one of the most important research topics in differential geometry. Research in recent years has shown that the issue of curves has an important place in other disciplines. Curves have become indispensable for research topics, especially in areas such as engineering and science. For example, as in studies $[6,8]$, curve theory was used in subjects such as waves in physics and cell modeling in biology. In this study, the Smarandache curve, which is a special curve in curve theory, has been studied. In 1909, the Salkowski curve is defined by E. Salkowski as a family of curves whose curvature is constant but the torsion is not constant [11]. In literature, these curves are known as Salkowski curves. The equation of the Salkowski curve was given by J. Monterde [9]. In Minkowski space, M. Turgut and S. Ylmaz described the Smarandache curves [14]. Later, according to the Darboux frame, Bishop frame and Sabban frame, some features of the Smarandache curves were investigated by $[3,4,15,5]$. The definition of timelike and spacelike Salkowski curve is given by 
A. T. Ali $[1,2]$. S. enyurt and K. Eren also studied the Smarandache curves obtained from the Frenet vectors of the spacelike Salkowski and anti-Salkowski curve [12, 13].

In this study, Smarandache curves are defined by a unit vector that is obtained from the linear combination of Frenet vectors of spacelike Salkowski curve. The Frenet apparatus of each curve are calculated and the graph of Smarandache curves is given.

\section{PRELIMINARIES}

Lorentzian inner product in the Minkowski space $R_{1}^{3}$ is defined by

$$
\langle,\rangle_{L}=d u_{1}^{2}+d u_{2}^{2}-d u_{3}^{2}
$$

where $u=\left(u_{1}, u_{2}, u_{3}\right) \in R_{1}^{3}$. The vector product of $u=\left(u_{1}, u_{2}, u_{3}\right)$ and $\mathrm{v}=\left(v_{1}, v_{2}, v_{3}\right)$ in $R_{1}^{3}$ is given by

$$
u \wedge v=-\left|\begin{array}{ccc}
i & j & -k \\
u_{1} & u_{2} & u_{3} \\
v_{1} & v_{2} & v_{3}
\end{array}\right|
$$

For $u \in R_{1}^{3}$, if $\langle u, u\rangle_{L}>0$ or $u=0$, then $u$ is spacelike vector, if $\langle u, u\rangle_{L}<0$, then $u$ is timelike vector, if $\langle u, u\rangle_{L}=0, u \neq 0$, then $u$ is lightlike (or null) vector. The norm of $u \in R_{1}^{3}$ is $\|u\|=\sqrt{\left|\langle u, u\rangle_{L}\right|}$. In [10], the Frenet vectors and the curvatures of the non-unit speed spacelike curve with a timelike principal normal $\gamma$ are

$$
\begin{array}{cl}
T(y)=\frac{\gamma^{\prime}(y)}{\left\|\gamma^{\prime}(y)\right\|}, \quad B(t)=\frac{\gamma^{\prime}(y) \wedge \gamma^{\prime \prime}(y)}{\left\|\gamma^{\prime}(y) \wedge \gamma^{\prime \prime}(y)\right\|}, \quad N(y)=B(y) \wedge T(y), \\
\kappa(y)=\frac{\left\|\gamma^{\prime}(y) \wedge \gamma^{\prime \prime}(y)\right\|}{\left\|\gamma^{\prime}(y)\right\|^{3}}, & \tau(y)=\frac{\left\langle\gamma^{\prime}(y) \wedge \gamma^{\prime \prime}(y), \gamma^{\prime \prime \prime}(y)\right\rangle}{\left\|\gamma^{\prime}(y) \wedge \gamma^{\prime \prime}(y)\right\|^{2}}, \\
& T^{\prime}=\kappa N, \\
& N^{\prime}=\kappa T+\tau B, \\
& B^{\prime}=\tau B .
\end{array}
$$

Definition 2.1. Let $\gamma$ and $\tilde{\gamma}$ be any two curves with Frenet frame $\{T, N, B\}$ and $\{\tilde{T}, \tilde{N}, \tilde{B}\}$, respectively. If $\langle T, \tilde{T}\rangle=0$, the curve $\tilde{\gamma}$ is called involute of the curve $\gamma$ and the curve $\gamma$ is called evolute of the curve $\tilde{\gamma}[7]$.

Definition 2.2. For $m>1$ or $m<-1$ and an arbitrary $m \in R$, let's define the space curve as follows

$$
\gamma_{m}(y)=\frac{n}{4 m}\left(\begin{array}{l}
2 \sin (y)-\sin ((1-2 n) y) \frac{1+n}{1-2 n}-\sin ((1+2 n) y) \frac{1-n}{1+2 n} \\
2 \cos (y)-\cos ((1-2 n) y) \frac{1+n}{1-2 n}-\cos ((1+2 n) y) \frac{1-n}{1+2 n} \\
\cos (2 n y) \frac{1}{m}
\end{array}\right)
$$

where $n=\frac{m}{\sqrt{m^{2}-1}}$. This curve is called the spacelike Salkowski curve. (Figure 1). The first curvature and the second curvature of Salkowski curve are $\kappa(y)=1$ and $\tau(y)=\cot (n y)$, respectively. 
In the work [2], the Frenet frame of spacelike Salkowski curve is given as following:

$$
\begin{aligned}
& T(y)=\left(\begin{array}{l}
\sin (n y) \cos (y)-n \sin (y) \cos (n y), \\
-\sin (y) \sin (n y)-n \cos (y) \cos (n y),-\cos (n y) \frac{n}{m}
\end{array}\right), \\
& N(y)=(\sin (y), \cos (y), m) \frac{n}{m}, \\
& B(y)=\left(\begin{array}{l}
-\cos (y) \cos (n y)-n \sin (y) \sin (n y), \\
\sin (y) \cos (n y)-n \cos (y) \sin (n y),-\sin (n y) \frac{n}{m}
\end{array}\right) .
\end{aligned}
$$

After this definition, the equation (2.2) is

$$
T^{\prime}=N, N^{\prime}=T+\tau B, B^{\prime}=\tau B .
$$

\section{Smarandache Curves Constructed from A Spacelike Salkowski Curve}

In this chapter, we describe the Smarandache curves constructed from a spacelike Salkowski curve and we calculate Frenet apparatus of the Smarandache curves.

Definition 3.1. Let $\gamma_{m}$ be a non planar spacelike Salkowski curve. The Smarandache curve constructed from a spacelike Salkowski curve $\gamma_{m}$ is defined by frame vectors as follows (Figure 2):

$$
\beta_{1}(y)=T(y)+N(y) .
$$

Substituting $T$ and $N$ vectors into the equation ( 2. 4 ), we get the curve $\beta_{1}(y)$ as follow:

$$
\beta_{1}(y)=\left(\begin{array}{l}
\sin (n y) \cos (y)-\sin (y) \cos (n y) n+\sin (y) \frac{n}{m}, \\
-\sin (y) \sin (n y)-\cos (y) \cos (n y) n+\cos (y) \frac{n}{m}, \\
-\cos (n y) \frac{n}{m}+n
\end{array}\right) .
$$

Theorem 3.2. Let $\gamma_{m}$ be a non planar spacelike Salkowski curve with Frenet apparatus $\{T, N, B, \kappa, \tau\}$ and $\beta_{1}$ be a Smarandache curve constructed from the curve $\gamma_{m}$, then Frenet aparatus of the curve $\beta_{1}$ is given by the followings:

$$
\begin{aligned}
& T_{T N}=\frac{T+N+\tau B}{|\tau|}, \\
& N_{T N}=\frac{\left(\tau^{\prime}-\tau\right) T+\left(\tau^{\prime}-\tau^{3}-\tau\right) N-\tau^{2} B}{\tau \sqrt{\left|\tau\left(\tau^{3}-2 \tau^{\prime}+\tau\right)\right|}}, \\
& B_{T N}=\frac{\left(\tau^{3}-\tau^{\prime}\right) T-\tau^{\prime} N-\tau^{2} B}{|\tau| \sqrt{\left|\tau\left(\tau^{3}-2 \tau^{\prime}+\tau\right)\right|}},
\end{aligned}
$$

where $\tau \neq 0, \tau^{3}-2 \tau^{\prime}+\tau \neq 0$ and $\kappa=1$.

Proof. Considering ( 2. 5 ), the derivate of the equation ( 3.6 ) is

$$
\beta_{1}{ }^{\prime}(y)=T+N+\tau B \text {. }
$$

The norm of this equation is

$$
\left\|\beta_{1}{ }^{\prime}(y)\right\|=|\tau| .
$$

From the equations ( 3.8 ) and (3.9), the tangent vector $T_{T N}$ of the Smarandache curve $\beta_{1}$ is obtained by

$$
T_{T N}=\frac{T+N+\tau B}{|\tau|}, \tau \neq 0 .
$$

If we take derivate the equation ( 3.8 ), we get

$$
\beta_{1}^{\prime \prime}(y)=T+\left(\tau^{2}+1\right) N+\left(\tau+\tau^{\prime}\right) B .
$$


From the equations ( 3.8 ) and ( 3.11 ), we find

$$
\beta_{1}^{\prime}(y) \wedge \beta_{1}^{\prime \prime}(y)=\left(\tau^{3}-\tau^{\prime}\right) T-\tau^{\prime} N-\tau^{2} B .
$$

The norm of the equation ( 3.12 ) is

$$
\left\|\beta_{1}{ }^{\prime}(y) \wedge \beta_{1}^{\prime \prime}(y)\right\|=|\tau| \sqrt{\left|\tau\left(\tau^{3}-2 \tau^{\prime}+\tau\right)\right|} .
$$

From the equations ( 3.12 ) and ( 3.13 ), the binormal vector $B_{T N}$ of the Smarandache curve $\beta_{1}$ is given

$$
B_{T N}=\frac{\left(\tau^{3}-\tau^{\prime}\right) T-\tau^{\prime} N-\tau^{2} B}{|\tau| \sqrt{\left|\tau\left(\tau^{3}-2 \tau^{\prime}+\tau\right)\right|}}, \tau \neq 0, \tau^{3}-2 \tau^{\prime}+\tau \neq 0 .
$$

From the equations ( 3.10 ) and (3.14), the principal normal vector $N_{T N}$ of the Smarandache curve $\beta_{1}$ is found

$$
N_{T N}=\frac{\left(\tau^{\prime}-\tau\right) T+\left(\tau^{\prime}-\tau^{3}-\tau\right) N-\tau^{2} B}{\tau \sqrt{\left|\tau\left(\tau^{3}-2 \tau^{\prime}+\tau\right)\right|}}, \tau \neq 0, \tau^{3}-2 \tau^{\prime}+\tau \neq 0 .
$$

Theorem 3.3. Let $\gamma_{m}$ be a non planar spacelike Salkowski curve with Frenet apparatus $\{T, N, B, \kappa, \tau\}$, then the first curvature and the second curvature of the Smarandache curve $\beta_{1}$ constructed from a spacelike Salkowski curve $\gamma_{m}$ are

$$
\kappa_{T N}=\frac{\sqrt{\left|\tau\left(\tau^{3}-2 \tau^{\prime}+\tau\right)\right|}}{\tau^{2}} \text { and } \tau_{T N}=\frac{3 \tau^{\prime 2}-\tau^{\prime} \tau-\tau^{\prime \prime} \tau}{\tau\left|\tau\left(\tau^{3}-2 \tau^{\prime}+\tau\right)\right|},
$$

where $\tau \neq 0, \tau^{3}-2 \tau^{\prime}+\tau \neq 0$ and $\kappa=1$.

Proof. Considering the equations ( 2.1 ), ( 3.9 ) and ( 3.13 ), the curvature $\kappa_{T N}$ of the Smarandache curve $\beta_{1}$ is found

$$
\kappa_{T N}=\frac{\sqrt{\left|\tau\left(\tau^{3}-2 \tau^{\prime}+\tau\right)\right|}}{\tau^{2}}, \tau \neq 0 .
$$

If we take derivate the equation ( 3.11 ), we get

$$
\beta_{1}^{\prime \prime \prime}(y)=\left(1+\tau^{2}\right) T+\left(\tau^{2}+3 \tau \tau^{\prime}+1\right) N+\left(\tau^{3}+\tau+\tau^{\prime}+\tau^{\prime \prime}\right) B .
$$

Considering the equations ( 3.8), (3.11), ( 3.13) and (3.17), the torsion $\tau_{T N}$ of the Smarandache curve $\beta_{1}$ is obtained by

$$
\tau_{T N}=\frac{3{\tau^{\prime}}^{2}-\tau^{\prime} \tau-\tau^{\prime \prime} \tau}{\tau\left|\tau\left(\tau^{3}-2 \tau^{\prime}+\tau\right)\right|}, \tau \neq 0, \tau^{3}-2 \tau^{\prime}+\tau \neq 0 .
$$

Definition 3.4. Let $\gamma_{m}$ be a non planar spacelike Salkowski curve with Frenet apparatus $\{T, N, B, \kappa, \tau\}$, then the Smarandache curve constructed from a spacelike Salkowski curve $\gamma_{m}$ is defined by frame vectors as follows (Figure 3):

$$
\beta_{2}(y)=\frac{T(y)+B(y)}{\sqrt{2}} .
$$


Substituting $T$ and $B$ vectors into the equation ( 2. 4 ), we get the curve $\beta_{2}$ as following:

$$
\beta_{2}(y)=\frac{1}{\sqrt{2}}\left(\begin{array}{l}
\cos (y)(\sin (n y)-\cos (n y)) \\
-n \sin (y)(\cos (n y)+\sin (n y)), \\
-\sin (y)(\sin (n y)-\cos (n y)) \\
-n \cos (y)(\cos (n y)+\sin (n y)), \\
-(\cos (n y)+\sin (n y)) \frac{n}{m}
\end{array}\right) .
$$

Theorem 3.5. Let $\gamma_{m}$ be a non planar spacelike Salkowski curve with Frenet apparatus $\{T, N, B, \kappa, \tau\}$ and $\beta_{2}$ be a the Smarandache curve constructed from the curve $\gamma_{m}$, then Frenet aparatus of the curve $\beta_{2}$ is given by the followings:

$$
T_{T B}= \pm N, N_{T B}= \pm \frac{T+\tau B}{\sqrt{\tau^{2}+1}}, B_{T B}=\frac{-\tau T+B}{\sqrt{\tau^{2}+1}}
$$

where $\kappa=1$.

Proof. Considering ( 2. 5 ) in derivate of the equation ( 3.18 ), we get

$$
\beta_{2}^{\prime}(y)=\frac{(1+\tau) N}{\sqrt{2}} \text {. }
$$

The norm of this equation is

$$
\left\|\beta_{2}^{\prime}(y)\right\|=\frac{|1+\tau|}{\sqrt{2}} .
$$

From the equations ( 3. 20 ) and (3. 21 ), the tangent vector $T_{T B}$ of the Smarandache curve $\beta_{2}$ is found as

$$
T_{T B}= \pm N
$$

If we take derivate the equation ( 3.20 ), we find

$$
\beta_{2}^{\prime \prime}(y)=\frac{(1+\tau) T+\tau^{\prime} N+\tau(1+\tau) B}{\sqrt{2}} .
$$

From the equations ( 3.20 ) and ( 3.23 ), we get

$$
\beta_{2}^{\prime}(y) \wedge \beta_{2}^{\prime \prime}(y)=\frac{(1+\tau)^{2}(-\tau T+B)}{2} .
$$

The norm of the equation ( 3.24 ) is obtained by

$$
\left\|\beta_{2}{ }^{\prime}(y) \wedge \beta_{2}{ }^{\prime \prime}(y)\right\|=\frac{(\tau+1)^{2} \sqrt{\tau^{2}+1}}{2} .
$$

From the equations ( 3.24 ) and ( 3.25 ), the binormal vector $B_{T B}$ of the Smarandache curve $\beta_{2}$ is found as

$$
B_{T B}=\frac{-\tau T+B}{\sqrt{\tau^{2}+1}}
$$

and from the equations ( 3.22 ) and (3.26), the principal normal vector $N_{T B}$ of the curve $\beta_{2}$ is obtained by

$$
N_{T B}= \pm \frac{T+\tau B}{\sqrt{\tau^{2}+1}}
$$


Theorem 3.6. Let $\gamma_{m}$ be a non planar spacelike Salkowski curve with Frenet apparatus $\{T, N, B, \kappa, \tau\}$, then the curvature and the torsion of the Smarandache curve $\beta_{2}$ constructed from a spacelike Salkowski curve $\gamma_{m}$ are

$$
\kappa_{T B}=\frac{\sqrt{2} \sqrt{\tau^{2}+1}}{|\tau+1|} \text { and } \tau_{T B}=\frac{\sqrt{2} \tau^{\prime}}{(\tau+1)\left(\tau^{2}+1\right)},
$$

where $\tau \neq-1$ and $\kappa=1$.

Proof. Considering the equations ( 3. 21 ), ( 3.25 ) and (2. 1 ), the curvature $\kappa_{T N}$ of the Smarandache curve $\beta_{2}$ is

$$
\kappa_{T B}=\frac{\sqrt{2} \sqrt{\tau^{2}+1}}{|\tau+1|}, \tau \neq-1 .
$$

If we take derivate the equation ( 3.23 ), we found

$$
\beta_{2}{ }^{\prime \prime \prime}(y)=\frac{2 \tau^{\prime} T+\left(\tau^{3}+\tau^{2}+\tau+1+\tau^{\prime \prime}\right) N+\tau^{\prime}(1+3 \tau) B}{\sqrt{2}} .
$$

From the equations ( 3. 20 ), (3. 23 ), ( 3. 25 ) and (3.29), the torsion of the Smarandache curve $\beta_{2}$ is obtained by

$$
\tau_{T B}=\frac{\sqrt{2} \tau^{\prime}}{(\tau+1)\left(\tau^{2}+1\right)}, \tau \neq-1
$$

Definition 3.7. Let $\gamma_{m}$ be a non planar spacelike Salkowski curve with Frenet apparatus $\{T, N, B, \kappa, \tau\}$, then the Smarandache curve constructed from a spacelike Salkowski curve $\gamma_{m}$ is defined by frame vectors as follows (Figure 4):

$$
\beta_{3}(y)=N(y)+B(y) \text {. }
$$

Substituting $N$ and $B$ vectors into the equation ( 2. 4 ), we get the curve $\beta_{3}$ as following:

$$
\beta_{3}(y)=\left(\begin{array}{c}
-\cos (y) \cos (n y)-\sin (y) \sin (n y) n+\sin (y) \frac{n}{m}, \\
\sin (y) \cos (n y)-\sin (n y) \cos (y) n+\cos (y) \frac{n}{m}, \\
n-\sin (n y) \frac{n}{m}
\end{array}\right) .
$$

Theorem 3.8. Let $\gamma_{m}$ be a non planar spacelike Salkowski curve with Frenet apparatus $\{T, N, B, \kappa, \tau\}$ and $\beta_{3}$ be a Smarandache curve constructed from the curve $\gamma_{m}$, then Frenet aparatus of the curve $\beta_{3}$ is given by the followings:

$$
\begin{aligned}
& T_{N B}=T+\tau N+\tau B, \\
& N_{N B}=-\frac{\tau T+\left(\tau^{2}+\tau^{\prime}+1\right) N+\left(\tau^{2}+\tau^{\prime}\right) B}{\sqrt{\left|\tau^{2}+2 \tau^{\prime}+1\right|}}, \\
& B_{N B}=\frac{\tau T-\tau^{\prime} N-\left(1+\tau^{\prime}\right) B}{\sqrt{\left|\tau^{2}+2 \tau^{\prime}+1\right|}},
\end{aligned}
$$

where $\tau^{2}+2 \tau^{\prime}+1 \neq 0$ and $\kappa=1$.

Proof. If we take derivate the equation ( 3.30 ), we obtain

$$
\beta_{3}{ }^{\prime}(y)=T+\tau N+\tau B .
$$

The norm of this equation is found as

$$
\left\|\beta_{3}{ }^{\prime}(y)\right\|=1 .
$$


From the equations ( 3.32 ) and ( 3.33 ), the tangent vector $T_{N B}$ of the Smarandache curve $\beta_{3}$ is

$$
T_{N B}=T+\tau N+\tau B .
$$

If we take derivate the equation ( 3.32 ), we have

$$
\beta_{3}^{\prime \prime}(y)=\tau T+\left(\tau^{2}+\tau^{\prime}+1\right) N+\left(\tau^{\prime}+\tau^{2}\right) B .
$$

From the equations ( 3.32 ) and ( 3.35 ), we found

$$
\beta_{3}{ }^{\prime}(y) \wedge \beta_{3}{ }^{\prime \prime}(y)=\tau T-\tau^{\prime} N-\left(1+\tau^{\prime}\right) B .
$$

The norm of the equation $(3.36)$ is

$$
\left\|\beta_{3}{ }^{\prime}(y) \wedge \beta_{3}{ }^{\prime \prime}(y)\right\|=\sqrt{\left|\tau^{2}+2 \tau^{\prime}+1\right|} .
$$

From the equations ( 3.36 ) and ( 3.37 ), the binormal vector $B_{N B}$ of the Smarandache curve $\beta_{3}$ is found by

$$
B_{N B}=\frac{\tau T-\tau^{\prime} N-\left(\tau^{\prime}+1\right) B}{\sqrt{\left|\tau^{2}+2 \tau^{\prime}+1\right|}}, \tau^{2}+2 \tau^{\prime}+1 \neq 0 .
$$

From the equations ( 3.34 ) and ( 3.38 ), the principal normal vector $N_{N B}$ of the curve $\beta_{3}$ is obtained

$$
N_{N B}=-\frac{\tau T+\left(\tau^{2}+\tau^{\prime}+1\right) N+\left(\tau^{2}+\tau^{\prime}\right) B}{\sqrt{\left|\tau^{2}+2 \tau^{\prime}+1\right|}}, \tau^{2}+2 \tau^{\prime}+1 \neq 0 .
$$

Theorem 3.9. Let $\gamma_{m}$ be a non planar spacelike Salkowski curve with Frenet apparatus $\{T, N, B, \kappa, \tau\}$, then the curvature and the torsion of the Smarandache curve $\beta_{3}$ constructed from a spacelike Salkowski curve $\gamma_{m}$ are

$$
\begin{aligned}
& \kappa_{N B}=\sqrt{\left|\tau^{2}+2 \tau^{\prime}+1\right|}, \\
& \tau_{N B}=\frac{\tau\left(\tau^{2}+1+2 \tau^{\prime}\right)-\tau^{\prime}\left(\tau^{3}+2+3 \tau \tau^{\prime}+\tau^{\prime \prime}\right)-\left(1+\tau^{\prime}\right)\left(\tau^{2}+1+2 \tau \tau^{\prime}+\tau^{\prime \prime}+\tau^{\prime}\right)}{\left|\tau^{2}+2 \tau^{\prime}+1\right|},
\end{aligned}
$$

where $\tau^{2}+2 \tau^{\prime}+1 \neq 0$ and $\kappa=1$.

Proof. Considering the equations ( 2. 1), ( 3.33 ) and (3. 37 ), the curvature of the curve $\beta_{3}$ is found by

$$
\kappa_{N B}=\sqrt{\left|\tau^{2}+2 \tau^{\prime}+1\right|}
$$

If we take derivate the equation ( 3.35 ), we get

$$
\begin{aligned}
& \gamma^{\prime \prime \prime}{ }_{N B}(y)=\left(\tau^{2}+1+2 \tau^{\prime}\right) T+\left(\tau^{3}+\tau+3 \tau \tau^{\prime}+\tau^{\prime \prime}\right) N \\
& +\left(\tau^{3}+1+2 \tau \tau^{\prime}+\tau^{\prime \prime}+\tau^{\prime}\right) B .
\end{aligned}
$$

From the equations ( 3.32$),(3.35),\left(3.37\right.$ ) and ( 3.42 ), the torsion of the curve $\beta_{3}$ is obtained by

$$
\begin{aligned}
& \tau_{N B}=\frac{\tau\left(\tau^{2}+1+2 \tau^{\prime}\right)-\tau^{\prime}\left(\tau^{3}+2+3 \tau \tau^{\prime}+\tau^{\prime \prime}\right)-\left(1+\tau^{\prime}\right)\left(\tau^{2}+1+2 \tau \tau^{\prime}+\tau^{\prime \prime}+\tau^{\prime}\right)}{\left|\tau^{2}+2 \tau^{\prime}+1\right|}, \\
& \tau^{2}+2 \tau^{\prime}+1 \neq 0 .
\end{aligned}
$$

Definition 3.10. Let $\gamma_{m}$ be a non planar spacelike Salkowski curve with Frenet apparatus $\{T, N, B, \kappa, \tau\}$, then the Smarandache curve constructed from a spacelike Salkowski curve $\gamma_{m}$ is defined by frame vectors as follows (Figure 5):

$$
\beta_{4}(y)=T(y)+N(y)+B(y) .
$$


Substituting $T, N$ and $B$ vectors into the equation (2. 4 ), we get the curve $\beta_{4}$ as following:

$$
\beta_{4}(y)=\left(\begin{array}{l}
\cos (y)(\sin (n y)-\cos (n y))-n \sin (y)(\cos (n y)+\sin (n y)) \\
+\sin (y) \frac{n}{m} \\
\sin (y)(\cos (n y)-\sin (n y))-n \cos (y)(\cos (n y)+\sin (n y)) \\
+\cos (y) \frac{n}{m} \\
n-\frac{n}{m}(\cos (n y)+\sin (n y))
\end{array}\right) .
$$

Theorem 3.11. Let $\gamma_{m}$ be a non planar spacelike Salkowski curve with Frenet apparatus $\{T, N, B, \kappa, \tau\}$ and $\beta_{4}$ be a the Smarandache curve constructed from the curve $\gamma_{m}$, then Frenet aparatus of the curve $\beta_{4}$ is given by the followings:

$$
\begin{aligned}
& T_{T N B}=\frac{T+(1+\tau) N+\tau B}{\sqrt{2|\tau|}}, \\
& N_{T N B}=\frac{(\tau+1) T+\left(\tau^{2}+1\right) N+\tau(\tau+1) B}{\sqrt{2|\tau|\left(\tau^{2}+1\right)}}, \\
& B_{T N B}=\frac{-\tau T+B}{\sqrt{\tau^{2}+1}},
\end{aligned}
$$

where $\tau \neq 0$ and $\kappa=1$.

Proof. If we take derivate the equation ( 3.43 ), we get

$$
\beta_{4}{ }^{\prime}(y)=T+(\tau+1) N+\tau B .
$$

The norm of this equation is

$$
\left\|\beta_{4}{ }^{\prime}(y)\right\|=\sqrt{2|\tau|}
$$

From the equations ( 3.45 ) and ( 3.46 ), the tangent vector $T_{T N B}$ of the Smarandache curve $\beta_{4}$ is found

$$
T_{T N B}=\frac{T+(1+\tau) N+\tau B}{\sqrt{2|\tau|}}, \tau \neq 0 .
$$

The derivate of the equation ( 3.45 ) is

$$
\beta_{4}^{\prime \prime}(y)=(\tau+1) T+\left(\tau^{2}+\tau^{\prime}+1\right) N+\tau(\tau+1) B
$$

From the equations ( 3.45 ) and ( 3.48 ), it is found

$$
\beta_{4}{ }^{\prime}(y) \wedge \beta_{4}^{\prime \prime}(y)=\left(2 \tau-\tau^{\prime}\right)(-\tau T+B) .
$$

The norm of this equation ( 3.49 ) is

$$
\left\|\beta_{4}{ }^{\prime}(y) \wedge \beta_{4}{ }^{\prime \prime}(y)\right\|=\left(2 \tau-\tau^{\prime}\right) \sqrt{\tau^{2}+1}
$$

From the equations ( 3.49 ) and ( 3.50 ), the binormal vector $B_{T N B}$ of the curve $\beta_{4}$ is found by

$$
B_{T N B}=\frac{-\tau T+B}{\sqrt{\tau^{2}+1}} .
$$

From the equations ( 3.47 ) and ( 3.51 ), the principal normal vector $N_{T N B}$ of the curve $\beta_{4}$ is obtained by

$$
N_{T N B}=\frac{(\tau+1) T+\left(\tau^{2}+1\right) N+\tau(\tau+1) B}{\sqrt{2|\tau|\left(\tau^{2}+1\right)}}, \tau \neq 0 .
$$


Theorem 3.12. Let $\gamma_{m}$ be a non planar spacelike Salkowski curve with Frenet apparatus $\{T, N, B, \kappa, \tau\}$, then the curvature and the torsion of the Smarandache curve $\beta_{4}$ constructed from a spacelike Salkowski curve $\gamma_{m}$ are

$$
\kappa_{T N B}=\frac{\left(2 \tau-\tau^{\prime}\right) \sqrt{\tau^{2}+1}}{|2 \tau|^{\frac{3}{2}}} \text { and } \tau_{T N B}=\frac{\tau^{\prime}(\tau+1)}{\left(2 \tau-\tau^{\prime}\right)\left(\tau^{2}+1\right)} \text {, }
$$

where $\tau \neq 0,2 \tau-\tau^{\prime} \neq 0$ and $\kappa=1$.

Proof. Considering the equations ( 2. 1 ), ( 3. 46 ) and ( 3. 50 ) the curvature of the Smarandache curve $\beta_{4}$ is found

$$
\kappa_{T N B}=\frac{\left(2 \tau-\tau^{\prime}\right) \sqrt{\tau^{2}+1}}{|2 \tau|^{\frac{3}{2}}}, \tau \neq 0 .
$$

The derivate of the equation ( 3.48 ) is

$$
\begin{aligned}
& \beta_{4}{ }^{\prime \prime \prime}(y)=\left(\tau^{2}+2 \tau^{\prime}+1\right) T+\left(\tau^{3}+\tau^{2}+\tau+3 \tau \tau^{\prime}+\tau^{\prime \prime}+1\right) N \\
& +\left(\tau^{3}+\tau+3 \tau \tau^{\prime}+\tau^{\prime}\right) B .
\end{aligned}
$$

From the equations ( 3.45 ), ( 3.48 ), ( 3.50 ) and ( 3.54 ), the torsion of the curve $\beta_{4}$ is obtained

$$
\tau_{T N B}=\frac{\tau^{\prime}(\tau+1)}{\left(2 \tau-\tau^{\prime}\right)\left(\tau^{2}+1\right)} .
$$

Corollary 3.13. The Smarandache curves $\beta_{1}$ and $\beta_{3}$ constructed from a spacelike Salkowski curve $\gamma_{m}$ are spacelike curves with a timelike principal normal.

Proof. Considering Theorems 3.2 and Theorem 3.8, proof is easily seen.

Corollary 3.14. The Smarandache curves $\beta_{2}$ and $\beta_{4}$ constructed from a spacelike Salkowski curve $\gamma_{m}$ are timelike curves.

Proof. Considering Theorem 3.5 and Theorem 3.11, proof is easily seen.

Corollary 3.15. The Smarandache curve $\beta_{2}$ is evolute of spacelike Salkowski curve with a timelike principal normal $\gamma_{m}$.

Proof. Considering the equations ( 2. 4 ) and (3. 22 ), we get $\left\langle T, T_{T B}\right\rangle=\langle T, N\rangle=0$. In that case, we call that the Smarandache curve $\beta_{2}$ is evolute of spacelike Salkowski curve $\gamma_{m}$.

\section{REFERENCES}

[1] A. T. Ali, Timelike Salkowski and anti-Salkowski curves in Minkowski 3- space, J. Adv. Res. Dyn. Cont. Syst., 2, (2010) 1726.

[2] A. T. Ali, Spacelike Salkowski and anti-Salkowski curves with timelike principal normal in Minkowski 3space, Mathematica Aeterna, 1, No. 4 (2011) 201-210.

[3] . Bekta and S. Yce, Special Smarandache curves according to Darboux frame in Euclidean 3-space, Romanian Journal of Mathematics and Computer sciencel, 3, No. 1 (2013) 48-59.

[4] M. etin, Y. Tuncer and M. K. Karacan, Smarandache curves according to Bishop frame in Euclidean 3-space, Gen. Math. Notes, 20, (2014) 50-66.

[5] A. alkan and S. enyurt, Smarandache curves in terms of Sabban frame of spherical indicatrix curves, Gen. Math. Notes, 31, No. 2 (2015) 1-15. 
[6] B. Ghanbari, S. Kumar and R. Kumar, A study of behaviour for immune and tumor cells in immunogenetic tumour model with non-singular fractional derivative, Chaos, Solitons \& Fractals, 133, (2020) 109619.

[7] H. H. Hacisalihoglu, Diferensiyel Geometry, Ankara University Faculty of Science Press, Ankara, 2000

[8] M. Jleli, S. Kumar, R. Kumar and B. Samet, Analytical approach for time fractional wave equations in the sense of Yang-Abdel-Aty-Cattani via the homotopy perturbation transform method, Alexandria Engineering Journal, 59, No. 5 (2020) 2859-2863.

[9] J. Monterde, Salkowski curves revisited: A family of curves with constant curvature and non-constant torsion, Computer Aided Geometric Design, 26, No. 3 (2009) 271-278.

[10] B. O’Neill, Semi-Riemannian Differential Geometry, Academic Press, USA, 1983.

[11] E. Salkowski, Zur transformation von Raumkurven, Math. Ann., 66, (1909) 517-557.

[12] S. enyurt and K. Eren, Smarandache curves of spacelike anti-Salkowski curve with a spacelike principal normal according to Frenet frame, GFBED/GUSTIJ, 10, No. 1 (2020) 251-260.

[13] S. enyurt and K. Eren, Smarandache curves of spacelike Salkowski curve with a spacelike principal normal according to Frenet frame. Erzincan University Journal of Science and Technology, 13, No. Special Issue -I (2020) 7-17.

[14] M. Turgut and S. Ylmaz, Smarandache curves in Minkowski spacetime, International J.Math. Combin., 3, (2008) 51-55.

[15] K. Takpr and M. Tosun, Smarandache curves on $S^{2}$, Boletim da Sociedade Paranaense de Matematica 3 Srie., 32, No. 1 (2014) 51-59. 

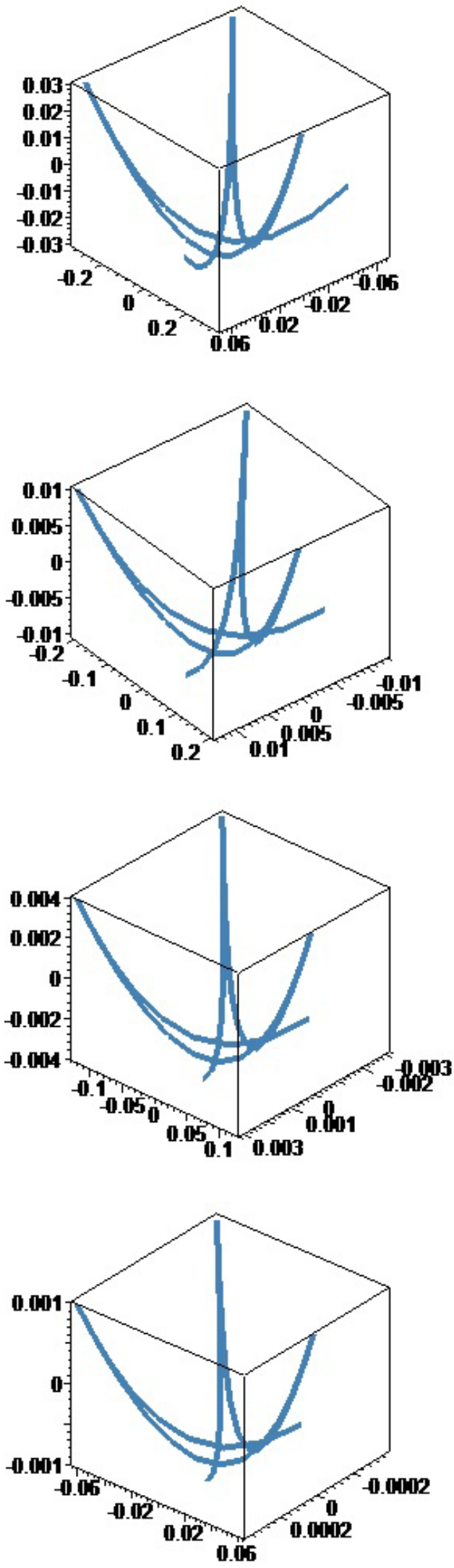

FIGURE 1. $m=\{3,5,8,16\}$ and $y \in[-5,5]$ for the spacelike Salkowski curve $\gamma_{m}$ 

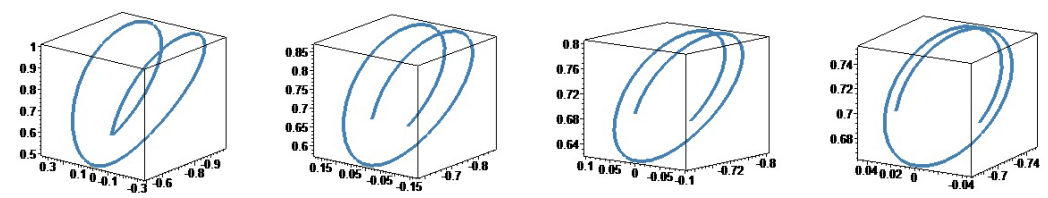

FIGURE 2. $m=\{3,5,8,16\}$ and $y \in[-5,5]$ for the Smarandache curve $\beta_{1}$
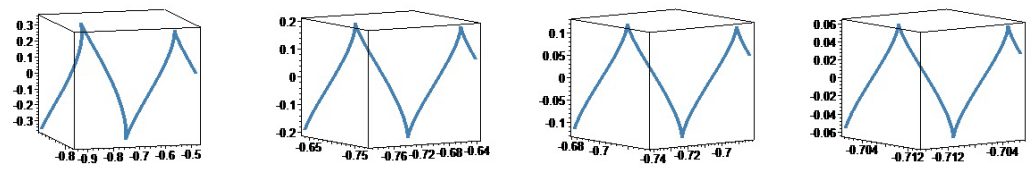

FIGURE 3. $m=\{3,5,8,16\}$ and $y \in[-5,5]$ for the Smarandache curve $\beta_{2}$
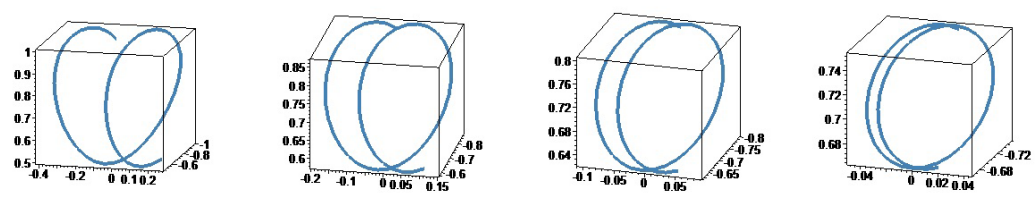

FIGURE 4. $m=\{3,5,8,16\}$ and $y \in[-5,5]$ for the Smarandache curve $\beta_{3}$
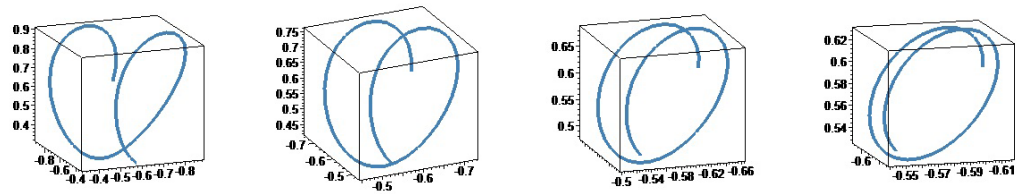

FIGURE 5. $m=\{3,5,8,16\}$ and $y \in[-5,5]$ for the Smarandache curve $\beta_{4}$ 